\title{
Lista de especies de peces en estadio larval en Bahía Portete (alta Guajira), Caribe colombiano
}

\author{
Checklist of larval fish species in Bahía Portete (Alta Guajira), Colombian \\ Caribbean
}

\author{
Juan J. Gallego, Andrés Cuellar y Alan Giraldo
}

\section{Resumen}

El conocimiento biológico de una región, además de tener gran importancia ecológica, es esencial para la conservación y aprovechamiento sostenible de los recursos naturales. En este estudio se presenta el listado taxonómico de las larvas de peces capturadas en aguas superficiales (1-10 m) de Bahía Portete, Guajira, Colombia durante 2 campañas oceanográficas realizadas en julio y noviembre de 2015. Se capturaron e identificaron un total de 2763 larvas de peces, pertenecientes a 37 familias y 66 especies. Durante julio, las especies con mayor frecuencia de captura fueron Anisotremus virginicus, Stegastes sp y Labrisomus sp; mientras que durante noviembre fueron Anchoa spinifer, Caranx sp.1 y Anisotremus virginicus. Estos resultados fortalecen las iniciativas de manejo integrado de los recursos naturales asociados a la zona costera de la alta Guajira e incrementan el conocimiento sobre la diversidad biológica marina del Parque Nacional Natural Portete-Kaurele y del Caribe colombiano.

Palabras clave. Colombia. Conservación. Diversidad. Ictioplancton. Parque Nacional Natural PorteteKaurele.

\begin{abstract}
Knowledge about the biological diversity of a region, in addition to its ecological importance, is essential for the conservation and sustainable use of natural resources. In this paper, we present a taxonomic list of fish larvae collected in surface waters $(1-10 \mathrm{~m})$ of Bahia Portete, Guajira, Colombia during 2 oceanographic campaigns in July and November 2015. A total of 2763 fish larvae were captured, belonging to 37 families and 66 species. During July, the species with higher frequency of captures were Anisotremus virginicus, Stegaste sp and Labrisomus sp; whereas during November the most frequent species were Anchoa spinifer, Caranx sp.1 and Anisotremus virginicus. These results strengthen initiatives of integrated management of natural resources in the coastal zone of the high Guajira and increase existing knowledge about marine biological diversity of the Natural National Park Portete-Kaurele and the Colombian Caribbean.
\end{abstract}

Keywords. Colombia. Conservation. Diversity. Ichthyoplankton. National Natural Park PorteteKaurele. 


\section{Introducción}

El conocimiento detallado de los grupos taxonómicos de una región, además de tener importancia ecológica, es esencial para la conservación y aprovechamiento sostenible de los recursos biológicos. En el caso de los peces, es de particular utilidad para determinar la oferta del recurso pesquero, evaluar la calidad ambiental y fortalecer acciones de manejo y conservación (Siqueiros-Beltrones y de la Cruz-Agüero, 2004; Aburto-Oropeza et al., 2011; Jupiter, et al. 2014). Particularmente, para el adecuado manejo del recurso íctico se hace necesario generar información científica pertinente que permita no solo conocer cómo responde el recurso en su estado adulto, sino la composición taxonómica, abundancia y variación espaciotemporal de los estadios iniciales de desarrollo, de tal manera que se pueda establecer de forma integral la dinámica poblacional de las especies de interés comercial o artesanal (Anderson et al., 2008; Hinrichensen et al., 2011; Zhou et al., 2011; Kough et al., 2013).

El ictioplancton está conformado por los huevos, larvas, postlarvas y en algunos casos juveniles de peces. Esto constituye un elemento primordial para comprender la biología, ecología y patrones de distribución de las especies de peces en su estado adulto, además de ser un elemento esencial en los procesos de flujo de energía y estabilidad de los ecosistemas acuáticos (Köster et al., 2001; Frederiksen et al., 2006; Montagnes et al., 2010; Zhou et al., 2011).

En su mayoría, los peces teleósteos liberan huevos flotantes o demersales que eclosionan en larvas vitelinas, transformándose en libres nadadores que se alimentan de pequeños organismos planctónicos (Chambers y Trippel, 1997; Kunz-Ramsay, 2004; Rodríguez et al., 2015). Todos estos estados de desarrollo se encuentran en la columna de agua principalmente sobre $200 \mathrm{~m}$ y son susceptibles de ser capturados por redes de plancton (Richards, 2006).

La información sobre el ictioplancton en el Caribe colombiano se remonta a los años 70, con el trabajo de Mercado (1970), quien realizó la descripción taxonómica de las larvas del sábalo (Megalops atlanticus) especie de importancia comercial, y Avila de Tabares (1978) quien realizó la descripción taxonómica del ictioplancton asociado a la ciénaga Grande de Santa Marta entre enero de 1970 y mayo de 1972. Posteriormente, el programa INPAVecep (1994 a 1999) generó la mayor cantidad de información científica del ictioplancton de la región (Manjarrés et al., 1994, 1997, 1998a, 1998b, 1998c; INPA, 1999; Vergara et al., 1999; Vergara y Arteaga, 1999), debido a que su propósito fundamental fue evaluar los recursos pesqueros presentes en la plataforma externa y el talud superior a lo largo del Caribe colombiano hasta la isóbata de $200 \mathrm{~m}$.

Aunque se han realizado otros estudios sobre ictioplancton en el Caribe colombiano (Escobar y Manjarrés, 1987; Rodríguez, 1996; Vergara, 1997; Medellín-Mora et al., 2013), la información disponible sobre estas fases de desarrollo de la fauna íctica en ambientes costeros de la zona norte del Caribe colombiano es escasa y más aún hacia el sector de la alta Guajira. En el presente trabajo se realizó la identificación taxonómica de las larvas de peces capturadas en Bahía Portete durante dos campañas de monitoreo oceanográfico, con el propósito de consolidar la lista de especies de peces que utilizan esta localidad como un sitio activo de su proceso reproductivo, generando así información que incrementa el conocimiento de la diversidad del componente íctico en esta área natural protegida de la región norte del Caribe colombiano.

\section{Materiales y métodos}

Bahía Portete se encuentra al norte del departamento de La Guajira, Caribe continental colombiano, entre el cabo de la Vela y Punta Gallinas, a los $12^{\circ} 12^{\prime} 56^{\prime \prime} \mathrm{N}-71^{\circ} 54^{\prime} 40^{\prime \prime} \mathrm{O}$ (Figura 1). Abarca una superficie aproximada de $125 \mathrm{~km}^{2}$, con $13 \mathrm{~km}$ de diámetro y se comunica con mar abierto a través de una boca de 2,1 km de ancho; 
presenta altos valores de salinidad (34-37 ups), temperatura $\left(25-30{ }^{\circ} \mathrm{C}\right)$ y turbidez en la columna de agua (1-4 m) y una concentración de oxígeno de 5-8 mg 1-1. Las condiciones climáticas locales están moduladas por el efecto de los vientos alisios del NE (Andrade, 1993; Solano, 1994).

Es una bahía somera, con un rango de profundidad entre $3 \mathrm{~m}$ y $20 \mathrm{~m}$, en donde se encuentran praderas de pastos marinos y bosques de manglar bordeando el margen sureste, y formaciones coralinas sobre los costados sur y occidental (Solano, 1994; INVEMAR, 2004; Gutiérrez-Moreno et al., 2008). En diciembre de 2014 esta área fue declarada Parque Nacional Natural por el Ministerio de Ambiente y Desarrollo Sostenible de la República de Colombia, teniendo como uno de los objetivos de conservación del área el "contribuir con la generación de servicios ecosistémicos que brindan los ecosistemas marítimos y costeros y sus especies asociadas, favoreciendo la productividad pesquera de la alta Guajira a través de la protección de espacios de incubación y crianza de juveniles de especies hidrobiológicas" (MADS, 2014).
Para obtener el listado taxonómico de las larvas de peces presentes en Bahía Portete se analizaron 84 muestras de zooplancton obtenidas durante dos campañas oceanográficas establecidas con base en los reportes locales disponibles de precipitación y vientos (Andrade, 1993; GuzmánAlvis, et al., 2006). La primera fue realizada en el mes de julio de 2015, momento que se caracteriza por una mayor influencia de los vientos alisios y menor precipitación promedio, y la segunda en noviembre de 2015, periodo inmediatamente posterior al momento de máxima precipitación reportada para esta localidad (Andrade, 1993; Guzmán-Alvis et al., 2006).

Las pescas de zooplancton se realizaron en 21 estaciones de muestreo distribuidas en toda la bahía, con el propósito de cubrir los hábitats submareales y litorales descritos para esta localidad, utilizando una red bongo de $30 \mathrm{~cm}$ de boca, equipada con una malla $300 \mu \mathrm{m}$ y una malla de $500 \mu \mathrm{m}$ de poro, mediante arrastres circulares superficiales diurnos (1-10 m), con duración de 10 minutos. Se utilizó un flujómetro Hydrobios ${ }^{\circledR}$

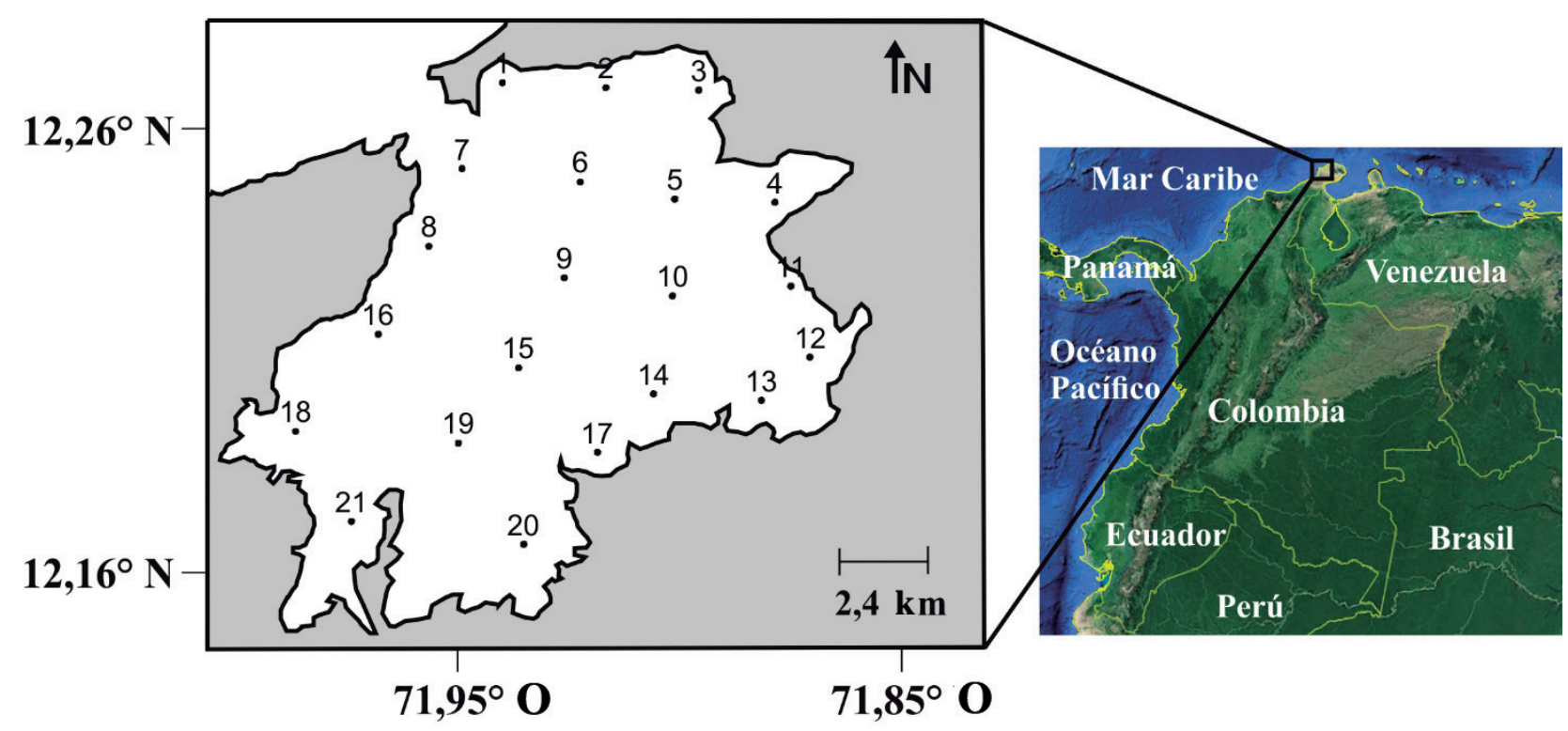

Figura 1. Ubicación de Bahía Portete en la alta Guajira, Caribe norte colombiano, indicando la ubicación de las 21 estaciones de muestreo durante dos campañas oceanográficas en julio y noviembre de 2015. Imagen fuente Digital Word ${ }^{\circledR}$ - Google Earth Pro ${ }^{\circledR}$. Fecha: 31 marzo 2012, Altura: 20 km, imagen centrada a $12^{\circ} 12^{\prime} 56^{\prime \prime} \mathrm{N}-71^{\circ}$. 
adosado a cada boca de la red para cuantificar el volumen de agua filtrada y estandarizar la abundancia por unidad de volumen de agua filtrada con el propósito de estimar la abundancia relativa de cada especie identificada.

Las muestras de zooplancton fueron fijadas en una solución de formalina-agua de mar a una concentración final de $4 \%$, y transportadas al laboratorio de Ecología Animal de la Universidad del Valle. En el laboratorio se revisó la totalidad de cada una de las muestras recolectadas y se separaron, contaron e identificaron hasta el menor nivel taxonómico posible todas las larvas de peces. Se utilizaron las guías de Beltrán y Herrera (2000) y Richards (2006) para realizar la identificación taxonómica. El material identificado fue depositado en la colección de referencia de Biología Marina de la Universidad del Valle (UNIVALLE: CIR-LV-UV: 0150001 \& 0150533).

\section{Resultados}

Se capturaron 2763 larvas de peces, pertenecientes a 37 familias y 66 especies (Anexo 1), de las cuales 1685 fueron capturadas en el mes de noviembre y 1078 en el mes de julio. En todas las estaciones de muestreo se encontraron larvas de peces, durante ambos periodos de muestreo. Durante julio, las familias con mayor frecuencia de captura fueron Haemulidae (95\%), Carangidae (76 \%) y Pomacentridae (95\%), mientras que durante noviembre fueron Carangidae (100\%), Haemulidae (90 \%) y Blenniidae (76 \%). Las especies con mayor frecuencia de captura durante julio fueron Anisotremus virginicus (Linnaeus, 1758), Stegastes sp. y Labrisomus sp.; y durante noviembre fueron Anchoa spinifer (Valenciennes, 1848), Caranx sp.1 y A. virginicus (Linnaeus, 1758) (Anexo 1).

Considerando ambos periodos de muestreo, las familias más abundantes fueron Haemulidae (24\%), seguida de Pomacentridae (17 \%) y Labrisomidae (12\%), siendo las especies más abundantes A. virginicus (21 \%) y Stegastes sp.
(17\%) (Anexo 1). De las 66 especies identificadas, el $38 \%$ correspondió a especies de interés para las actividades pesqueras, destacando por su abundancia Anisotremus virginicus (21\%) (Figura 2). La familia con mayor riqueza fue Carangidae, con un total de 10 especies identificadas, seguida por las familias Haemulidae y Blenniidae, con 4 especies cada una. Además, es importante destacar que el mayor número de especies se registró en el mes de noviembre con 57 especies, mientras que durante julio solo se registraron 37. Las especies de mayor interés comercial fueron Bairdiella ronchus (Cuvier, 1830), Balistes capriscus (Gmelin, 1789), Trachurus lathami (Nichols, 1920), Trichiurus lepturus (Linnaeus, 1758), Opisthonema oglinum (Lesueur, 1818), Sardinella brasiliensis (Steindachner, 1879) y Sardinella aurita (Valenciennes, 1847) (Figura 2).

\section{Discusión}

Los resultados del presente estudio son similares a los reportados en otros ecosistemas marinos y estuarios someros: densidades larvales relativamente bajas, una predominancia de larvas pequeñas y una baja diversidad (Houde y Foodal, 1984; Ditty, 1986; Powell et al., 1989; Vásquez-Yeomans y Richards, 1999). Estudios previos en la zona costera norte del Caribe colombiano reportaron que las familias de larvas de peces más abundantes son Gobiidae, Carangidae, Sciaenidae y Synodontidae, siendo las especies con mayor porcentaje de aparición Eucinostomus sp., Lutjanus synagris y Upeneus parvus (Manjarrés et al., 2005a, b). Estos autores realizaron el trabajo de identificación considerando las larvas capturadas con un ojo de malla de $500 \mu \mathrm{m}$. A diferencia de estos trabajos previos, la descripción de la composición de larvas de peces presentes en Bahía Portete se realizó a partir del análisis de las capturas realizadas con malla de 300 y $500 \mu \mathrm{m}$, lo que incrementó de manera sustancial la probabilidad de captura de larvas de un mayor número de familias como lo sugieren Jhon y Vernie (1996), Morse (1989) y Jennings (1991), estando dominado el 


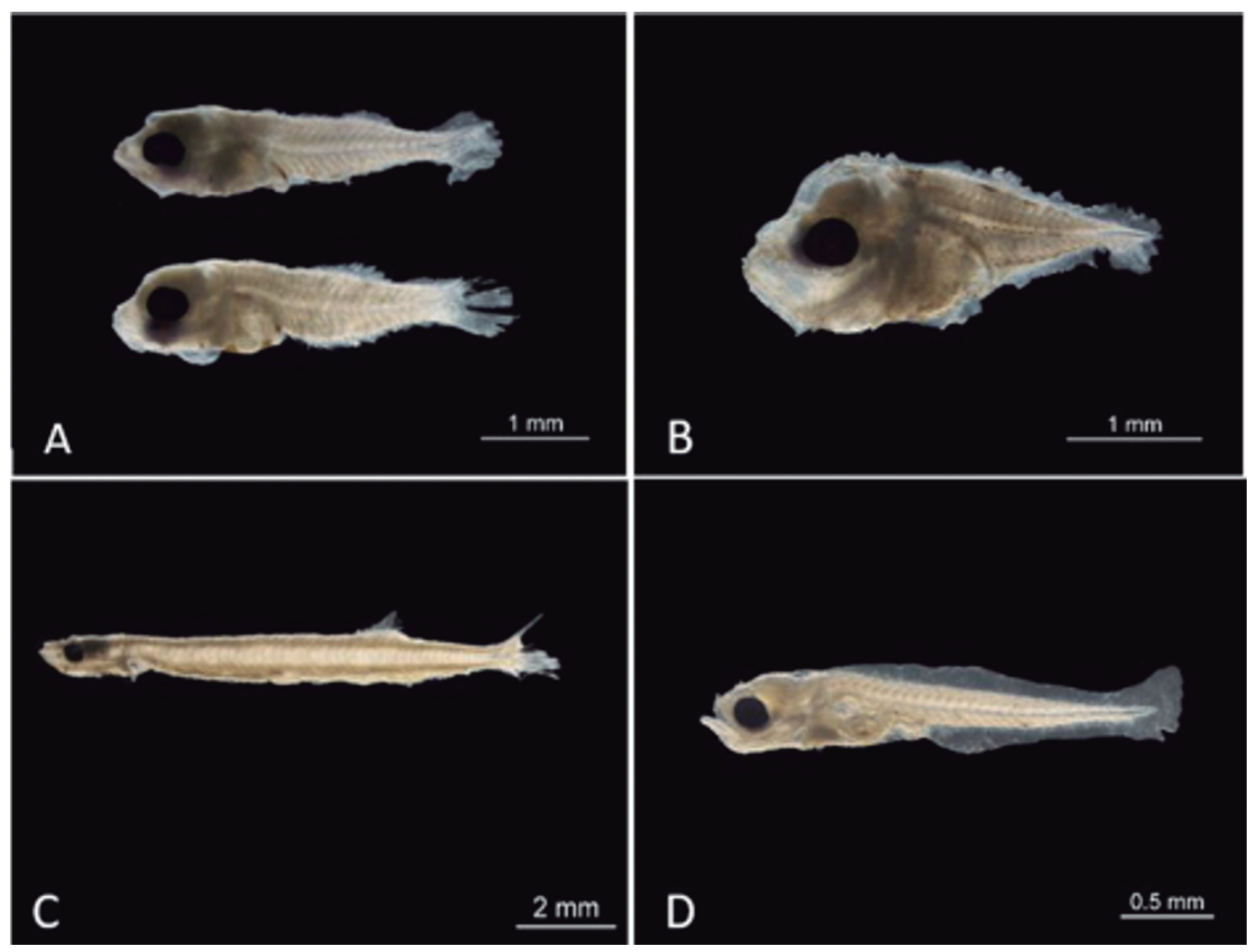

Figura 2. Larvas de peces de especies comerciales más abundantes. A) Anisotremus virginicus, B) Caranx sp. 1, C) Sardinella aurita, D) Trichiurus lepturus.

ictioplancton de esta localidad por cinco familias de peces (Haemulidae, Carangidae, Engraulidae, Labrisomidae y Pomacentridae), que juntas representaron el $72 \%$ del total de las larvas de peces identificadas.

Los peces demersales y pelágicos representan un importante recurso económico para las pesquerías artesanales del Caribe colombiano, lo que significa que son principales objetivos de captura (Correa y Manjarrés, 2004; Paramo et al., 2009; García, 2010). Entre estas especies se destacan los pargos (lutjánidos), las corvinas (Sciaenidae), los coroncoros o roncadores (haemúlidos) y los cachicachis (Sparidae) (Correa y Manjarrés, 2004; Viloria-Maestre et al., 2016). Sin embargo, en las últimas décadas se ha presentado una reducción significativa en la captura total reportada para esta región, llevando a esta actividad económica hacia niveles de subsistencia (García, 2010; Parraga et al., 2010). Por tal motivo, han sido incorporadas nuevas especies como objeto de pesca artesanal, como es el caso de Trichurus lepturus; especies que hasta hace algunos años era un acompañante de la pesca de pargos y roncos, siendo ahora uno de los peces de interés en la pesca artesanal del Caribe colombiano (Gómez, 2009; SIPA, 2010). En el presente estudio, no solo se registraron larvas de T. lepturus en Bahía Portete, sino de varias especies que son de interés para la pesca artesanal del Caribe colombiano, lo que pone de manifiesto la importancia de esta localidad como lugar de crianza para el recurso pesquero artesanal de la alta Guajira (Paramo et al., 2009; García, 2010).

Teniendo en cuenta la riqueza de especies que han sido reportadas en las aguas adyacentes al Caribe colombiano, así como el mayor esfuerzo de muestreo en ciertas áreas de la costa y el énfasis hacia grupos de importancia comercial, 
el conocimiento disponible sobre la composición del ictioplancton en el mar Caribe colombiano puede aún ser considerado como bajo (Medellín et al., 2013). Recientemente, Miloslavich et al. (2010) estimaron que el número de especies de peces que habitan en todo el mar Caribe es de 1336, de los cuales, de acuerdo con Polanco et al. (2010), el 82,3 $\%$ se encuentran presentes en el Caribe colombiano. Lamentablemente, solamente se tiene información disponible de los estadios larvales del 8,7 \% de las especies conocidas en estado adulto que habitan en esta región del Caribe (Polanco et al., 2010).

Una forma de incrementar el conocimiento acerca de los estados larvales de los peces presentes en una localidad es por medio de la consolidación de listados taxonómicos como el que se presenta en este trabajo. Este tipo de esfuerzo no solo incrementa la información general sobre una de las fases de desarrollo críticas para el componente íctico de una región, sino que también proporciona información relevante que puede ser utilizada en el marco de las acciones direccionadas a valorar y conservar la biodiversidad marina local (Warner et al., 2000). Además, uno de los insumos de conocimiento requeridos para comenzar a entender los patrones de distribución espacial, la variación temporal de la abundancia y las relaciones ecológicas con el hábitat disponible de los peces adultos, es conocer la identidad taxonómica de sus larvas y establecer como estas varían espacial y temporalmente (Hermes-Silva et al., 2009, Costa et al., 2015). Por lo tanto, los resultados obtenidos en la presente investigación son de particular importancia para fortalecer en el corto plazo las acciones de manejo de los recursos naturales asociados a la zona costera de la alta Guajira, incrementando simultáneamente el conocimiento sobre la diversidad biológica del recientemente declarado Parque Nacional Natural Portete-Kaurele.

\section{Agradecimientos}

Isabell Calle, Marisol Rivera y Mauricio Jerez por el apoyo durante las campañas de muestreo oceanográfico. A Gerardo Aceves Medina del Centro Interdisciplinario de Ciencias Marinas (CICIMAR-IPN), La Paz, Baja California Sur, México, por confirmar la identidad taxonómica de las larvas de peces. A María Claudia DíazGranados, coordinadora del programa marino de Conservación Internacional Colombia, por su apoyo incondicional en la gestión administrativa y logística requerida para el desarrollo de esta investigación. Este trabajo fue financiado parcialmente por el grupo de investigación en Ciencias Oceanográficas de la Universidad del Valle, el programa marino de Conservación Internacional Colombiana y Cerrejón Puerto Bolívar en el marco del Convenio Acuerdos de Pesca 2015. Las pescas de zooplancton en el PNN Portete-Kaurele estuvieron amparados por el permiso individual de recolección de especímenes de especies silvestres de la diversidad biológica con fines de investigación científica no comercial -PIDB DTCA No. 024-15, Resolución 183 de 24 de noviembre de 2015 del Ministerio de Ambiente y Desarrollo Sostenible, Parques Nacionales Naturales de Colombia.

\section{Referencias}

Aburto-Oropeza, O., Erisman, B., Galland, G. R., Mascareñas-Osorio, I., Sala, E. y Ezcurra, E. (2011). Large recovery of fish biomass in a notake marine reserve. PLoS ONE, 6(8), e23601. Doi: 10. 1371/journal.

Andrade, C. A. (1993). Análisis de la velocidad del viento sobre el Mar Caribe (Analysis of the surface-wind speed over the Caribbean Sea). Bol. Cient. $\mathrm{CIOH}, 13,33-44$.

Anderson, C. N. K., Hsieh, C., Sandin, S. A., Hewitt, R., Hollowed, A., Beddington, J., May, R. M. y Sugihara, G. (2008). Why fishing magnifies fluctuations in fish abundance. Nature, 452, 835839.

Avila de Tabarés, G. (1978). Ictioplancton de la ciénaga Grande de Santa Marta; enero 1970mayo 1972. INDERENA-Divulgación Pesquera 12(1). 
Beltrán, S. y Herrera, R. (2000). Estadios tempranos de peces del Pacífico Colombiano. 727 pp. Buenaventura, Instituto Nacional de Pesca y Acuicultura (INPA).

Chambers, R. C. y Trippel, E. (Eds.). (1997). Early life history and recruitment in fish populations. 595 pp. Londres, UK: Chapman \& Hall.

Correa, F. y Manjarrés, L. (2004). Inventario y caracterización general de las unidades económicas de pescas artesanales de La Guajira, Caribe colombiano. Pp. 77-93. En L. Manjarrés. Pesquerías demersales del área norte del Caribe colombiano y parámetros ecológicos, biológicos pesqueros y poblacionales del recurso pargo. Santa Marta, Colombia: Universidad del Magdalena.

Correa, F. y Manjarrés, L. (2004). Recursos pesqueros demersales explotados por las pesquerías artesanales marítimas de La Guajira, Caribe colombiano. Pp. 77-92.

Costa, M. D., Possinghamy, H. P. y Muelbert, J. H. (2015). Incorporating early life stages of fishes into estuarine spatial conservation planning. Aquatic Conservation: Marine and Freshwater Ecosystems, 26(6), 1013-1030.

Ditty, J. G. (1986). Ichthyoplankton in neritic waters of the northern Gulf of Mexico off Louisiana: composition, relative abundance, and seasonality. Fishery Bulletin. U.S, 84, 935-946.

Escobar, A. y Manjarrés, G. (1987). Consideraciones generales sobre el ictioplancton de la región de La Guajira, Colombia. Revista Ingeniería Pesquera, 7(1-2), 8-29.

Frederiksen, M., Edwards M., Richardson A. J., Halliday, N. C. y Wanless, S. (2006). From plankton to top predators: bottom-up control of a marine food web across four trophic levels. Journal of Animal Ecology, 75(6), 1259-1268.

Froese, R. y Pauly, D. (Eds.). (2017). FishBase. World Wide Web electronic publication. www. fishbase.org, versión (10/2017).

García, C. B. (2010). Conocimiento tradicional: lo que los pescadores artesanales del Caribe colombiano tienen para decirnos. Pan-American Journal of Aquatic Sciences, 5(1), 78-90.
Gómez, S. (2009). Biología reproductiva, captura por unidad de esfuerzo y estacionalidad de Trichurus lepturus (Linnaeus, 1758) (Trichiuridae: Teleostei) en la Bahía de Gaira, Caribe de Colombia (Trabajo de grado) (76 pp). Bogotá D. C.: Universidad Jorge Tadeo Lozano, Facultad de Ciencias Naturales, Departamento de Biología Marina.

Gutiérrez-Moreno, C., Alonso, D. y SeguraQuintero, C. (2008). Diseño de un área marina protegida para Bahía Portete-La Guajira, Caribe colombiano. Boletín de Investigaciones Marinas y Costeras-INVEMAR, 37(2), 189-212.

Guzmán-Alvis, A. I., Lattig, P. y Ruiz, J. A. (2006). Spatial and temporal characterization of soft bottom polychaetes in a shallow tropical bay (Colombian Caribbean). Boletín de Investigaciones Marinas y Costeras-INVEMAR, 35(1), 19-36.

Hermes-Silva, S., Reynalte-Tataje, D. A. y Zaniboni-Filho, E. (2009). Spatial and Temporal Distribution of Ichthyoplankton in the Upper Uruguay River, Brazil. Brazilian Archives of Biology and Technology, 52, 933-944.

Hinrichensen, H. H., Dickey-Collas, M., Huret, M., Peck, M. A. y Vikebø, F. B. (2011). Evaluating the suitability of coupled biophysical models for fishery management. ICES Journal of Marine Science, 68(7), 1478-1487.

Houde, E. D. y Lovdal, J. D. A. (1984). Seasonality of ocurrence foods and food preferences of ichthyoplankton in Biscayne Bay, Florida. Estuarine Coastal Shelf Science, 18, 403-419.

INPA. Instituto Nacional de Pesca y Acuicultura. (1999). Boletín estadístico pesquero 1997-1998, INPA. 118 pp. Bogotá D. C.

INVEMAR. (2004). Monitoreo de ecosistemas Representativos de Bahía Portete. 137 pp. Informe final. Santa Marta: INVEMAR.

Jennings, S. (1991). The effects of capture, net retention and preservation upon lengths of larval and juvenile bass, Dicentrarchus labrax (L.). Journal of Fish Biology, 38(3), 349-357.

John, A. y Vernie, J. (1966). The relationship between size of net used and estimates of zooplankton 
diversity. Limnology and Oceanography, 11(4), 456-469.

Jupiter, S. D., Cohen, P. J., Weeks R., Tawake, A. y Govan, H. (2014). Locally-managed marine areas: multiple objectives and diverse strategies. Pacific Conservation Biology, 20(2), 165-179.

Köster, F. W., Hinrichsen, H., John, M. A., Schnack, D., MacKenzie, B. R., Tomkiewicz, J. y Plikshs, M. (2001). Developing Baltic cod recruitment models. II. Incorporation of environmental variability and species interaction. Canadian Journal Fish Aquatic Science, 58(8), 1534-1556.

Kough, A. S., Paris, C. B. y Butler, IV M. J. (2013). Larval connectivity and the international management of fisheries. PLoS ONE, 8(6), e64970. doi: 10.1371 /journal.pone.0064970

Kunz-Ramsay, Y. (2004). Developmental biology of teleost fishes (637 pp). Springer Netherlands

MADS. (2014). Resolución 2096 de 19 de diciembre de 2014. Por medio de la cual se declara, reserva, delimita y alindera el Parque Nacional Natural Bahía Portete-Kaurele. Ministerios de Ambiente y Desarrollo Sostenible. 30 pp.

Manjarrés, L. (Ed). (2004). Pesquerías demersales del área norte del Mar Caribe de Colombia y parámetros biológico-pesqueros y poblacionales del recurso pargo. Santa Marta.

Manjarrés, L., Vergara, A., Torres, J., Rodríguez, G., Arteaga, E., Viaña, J., Arévalo, J. y Galvis R. (2005a). Evaluación de peces demersales $e$ ictioplancton en el mar caribe de Colombia, incluyendo condiciones oceanográficas. Parte I: Crucero INPAVECEP/UE/DEMER/9507 (51 pp). Santa Marta.

Manjarrés, L., Rodríjuez, G., Torres, J., Vergara, A., Arteaga, E., Arévalo, J., Galvis, R., Rodríguez, D. y Viana, J. (2005b). Evaluación de peces demersales e ictioplancton en el mar caribe de Colombia, incluyendo condiciones oceanográficas (87 pp). Parte II: Crucero INPA-VECEP/UE/DEMER/9507. Santa Marta.

Manjarrés, L., Torres, J., Vergara, A., Rodríguez, G., Viaña, J. y Correa, J. (1994). Crucero de evaluación de recursos demersales en el Caribe colombiano
(52 pp). VECEP/DEMER/9401. Informe final. Santa Marta: INPA.

Manjarrés, L., Torres, J., Rodríguez, G., Vergara, A., Arteaga, E., Viaña, J., Arévalo, J., Galvis, R., Rodríguez, J., Amaya, F., García, D., Páramo, J. y León, G. (1997). Crucero de evaluación de peces pelágicos pequeños en el Caribe colombiano (34 pp). Informe final. Santa Marta: Instituto Nacional de Pesca y Acuicultura (INPA).

Manjarrés, L., Torres, J., Rodríguez, G., Vergara, A., Arteaga, E., Viaña, J., Arévalo, J., Galvis, R., Rodríguez, J., Amaya, F., García, D., Páramo, J. y León, G. (1998a). Crucero de evaluación de peces pelágicos pequeños en el Caribe colombiano (47 pp). Informe final. Santa Marta: Instituto Nacional de Pesca y Acuicultura (INPA).

Manjarrés, L., Torres, J., Rodríguez, G., Vergara, A., Arteaga, E., Viaña, J., Arévalo, J., Galvis, R., Rodríguez, J., Amaya, F., García, D., Páramo, J. y León, G. (1998b). Campaña de evaluación de peces pelágicos pequeños en el Caribe colombiano. Informe final (57 pp). Santa Marta: Instituto Nacional de Pesca y Acuicultura (INPA).

Manjarrés, L., Torres, J., Rodríguez, G., Vergara, A., Arteaga, E., Viaña, J., Arévalo, J., Galvis, R., Rodríguez, J., Amaya, F., García, D., Páramo, J. y León, G. (1998c). Campaña de evaluación de peces pelágicos pequeños en el Caribe colombiano. (58 pp). Informe final. Santa Marta: Instituto Nacional de Pesca y Acuicultura (INPA).

Medellín-Mora, J., Polanco, A. y Navas, G. (2013). Inventario de larvas de peces registradas para el Caribe colombiano. Boletín de Investigaciones Marinas y Costeras, 42(2), 233-253.

Mercado, J. (1970). Notas sobre los estados larvales del sábalo, Megalops atlanticus, con comentarios sobre su importancia comercial y deportiva (Trabajo de grado) (32 pp). Cartagena, Colombia: Universidad Jorge Tadeo Lozano, Facultad de Ciencias, Departamento de Biología Marina.

Miloslavich, P., Díaz, J. M., Klein, E., Alvarado, J. J., Díaz, C., Gobin, J., Escobar-Briones, E., Cruz-Motta, J. J., Weil, E., Cortés, J., Bastidas, 
A. C., Robertson, R., Zapata, F., Martín, A., Castillo, J., Kazandjian, A. y Ortiz, M. (2010). Marine biodiversity in the Caribbean: Regional estimates and distribution patterns. PLOS ONE, 5(8), 1-25.

Montagnes, D. J., Dower, J. F. y Figueiredo, G. M. (2010). The protozooplankton-ichthyoplankton trophic link: an overlooked aspect of aquatic food webs. Journal of Eukaryotic Microbiology, $57(3), 223-228$.

Morse, W. W. (1989). Catchability, growth, and mortality of larval fishes. Fishery Bulletin, 87(3), 417-446.

Polanco, F., Acero, A. y Garrido, M. (2010). Aportes a la biodiversidad íctica del Caribe colombiano (316-353 pp). En Navas, G. R., Segura-Quintero C., Garrido-Linares M., Benavides-Serrato M. y Alonso D. (Eds.). Biodiversidad del margen continental del Caribe colombiano (458 pp). Santa Marta: Serie de publicaciones especiales No. 20, INVEMAR.

Paramo, J., Guillot-Illidge, L., Benavides, S., Rodríjuez, A., y Sánchez-Ramírez, C. (2009). Aspectos poblacionales y ecológicos de peces demersales de la zona norte del Caribe colombiano en relación con el hábitat: una herramienta para identificar Áreas Marinas Protegidas (AMPs) para el manejo pesquero. Caldasia, 31(1), 123-144.

Párraga, D. P., Cubillos, L. A. y Correa-Ramírez, M. A. (2010). Variaciones espacio-temporales de la captura por unidad de esfuerzo en la pesquería artesanal costera del pargo rayado Lutjanus synagris, en el Caribe colombiano y su relación con variables ambientales. Revista de biología marina y oceanografía, 45(1), 77-88.

Powell, A. B., Hoss, D. E., Hettler, W. F., Peters, D. S. y Wagner, S. (1989). Abundance and distribution of ichthyoplankton in Florida Bay and adjacent waters. Bulletin of Marine Science, 44, 35-48.

Richards, W. (2006). Early stages of Atlantic fishes: an identification guide for the Western Central North Atlantic (2640 pp).Vol. 1. Boca Ratón, EE. UU: Taylor y Francis Group, CRC Press.
Rodríguez, A., Rueda, M. y Escobar, F. (2015). Evaluación directa de las poblaciones de peces grandes pelágicos del Pacífico y Caribe continental de Colombia (120 pp). Santa Marta, Colombia: INVEMAR y AUNAP. Serie Recursos Pesqueros de Colombia- AUNAP 2015.

Rodríguez, J. (1996). Abundancia y distribución de larvas de peces demersales en el área suroccidental del Caribe colombiano (Trabajo de grado) (110 pp). Santa Marta: Universidad Jorge Tadeo Lozano, Facultad de Ciencias, Departamento de Biología Marina.

SIPA. (2010). El Sable (Trichiurus lepturus) en la pesca artesanal. Sistema de información de pesca y acuicultura. Boletín mensual, (49).

Siqueiros-Beltrones, D. A. y de la Cruz-Agüero, J. (2004). Examen filosófico de las listas sistemáticas como informes científicos y porqué deben ser publicados. Oceánides, 19, 1-9.

Solano, O. (1994). Corales, formaciones arrecifales y blanqueamiento de 1987 en Bahía Portete (La Guajira, Colombia). Anales de Instituto de Investigaciones Marinas de Punta Betín, 23, 149-163.

Vásquez-Yeomans, L. y Richards, W. J. (1999). Variación estacional del ictioplancton de la Bahía de la Ascensión, Reserva de la Biósfera de Sian Ka'an (1990-1994). Revista de Biología Tropical, 47, 201-212.

Vergara, A. (1997). Distribución, abundancia y diversidad de larvas de peces demersales comerciales en la zona nerítica frente al departamento de La Guajira, Caribe colombiano (Trabajo de grado) (171 pp). Universidad Nacional de Colombia, Santa Marta.

Vergara, A., y Arteaga, E. (1999). Abundancia y distribución de larvas de peces de las familias Carangidae, Clupeidae y Engraulidae en el nororiente del litoral Caribe colombiano. INPA Boletín Científico Suplemento, 6, 83-95.

Vergara, A., Arteaga, E. y Rodríguez, J. (1999). Composición abundancia y distribución del ictioplancton del Caribe colombiano. INPA Boletín Científico Suplemento, 6, 77-83. 
Viloria-Maestre E., Santos-Acevedo M., Chávez S., Romero J. A. (2016). Pesquería artesanal del margen costero entre Los Cocos (Magdalena) y Punta Gallinas (La Guajira), Caribe colombiano. Santa Marta: Serie dePublicaciones Generales del Invemar. No. 92, $66 \mathrm{pp}$.

Warner, R. R., Swearer, S. E. y Caselle, J. E. (2000). Larval accumulation and retention: implications for the design of marine reserves and essential habitat. Bulletin of Marine Science, 66(3), 821-830.

Zhou, M., Lin Y., Yang S., Cao W. y Zheng L. (2011). Composition and ecological distribution of ichthyoplankton in eastern Beibu Gulf. Acta Oceanológica Sinica, 30(1), 94-105.

Anexo 1. Lista taxonómica del ictioplancton capturado en Bahía Portete durante julio y noviembre de 2015. Disponible en línea: http:/ / revistas.humboldt.org.co/index.php/biota/rt/suppFiles/559/0

Juan José Gallego

Universidad del Valle,

Facultad de Ciencias Naturales y Exactas, Departamento de Biología, Grupo de investigación en Ciencias

Oceanográficas

Cali, Colombia

juan.j.gallego@correounivalle.edu.co

\section{Andrés Cuellar}

Universidad del Valle,

Facultad de Ciencias Naturales y Exactas, Departamento de Biología, Grupo de investigación en Ciencias

Oceanográficas

Cali, Colombia

andres.cuellar@correounivalle.edu.co

\section{Alan Giraldo}

Universidad del Valle,

Facultad de Ciencias Naturales y Exactas, Departamento de Biología, Grupo de investigación en Ciencias

Oceanográficas

Cali, Colombia

alan.giraldo@correounivalle.edu.co
Lista de especies de peces en estadio larval en Bahía Portete (alta Guajira), Caribe colombiano

Citación del artículo: Gallego, J. J., Cuellar, A. y Giraldo, A. (2018). Lista de especies de peces en estadio larval en Bahía Portete (alta Guajira), Caribe colombiano. Biota Colombiana, 19(1), 123132. DOI: $10.21068 / \mathrm{c} 2018 . v 19$ n01a08.

Recibido: 13 de marzo de 2017

Aprobado: 27 de marzo de 2018 